\title{
FORMAS DE CARBONO EM LATOSSOLO VERMELHO EUTROFÉRRICO SOB PLANTIO DIRETO NO SISTEMA BIOGE OGRÁFICO DO CERRADO(1)
}

\author{
M. E. C. ROSA ${ }^{(2)}$, N. OLSZEVSKI (3), E. S. MENDONÇA(4), \\ L. M. $\operatorname{COSTA}^{(4)} \&$ J . R. CORREIA(5)
}

\begin{abstract}
RESUMO
Este trabalho teve por objetivo avaliar aspectos da física, química e da dinâmica de carbono de um Latossolo Vermelho Eutroférrico, sob diferentes condições de uso. Foram utilizados materiais de solo cultivado com plantio di reto, irrigado e sequeiro, e sob floresta nativa, na região de Santa Helena de Goiás (GO). As amostras foram coletadas de camadas de 0,00-0,05, 0,05-0,10, 0,10-0,20, $0,20-0,30$ e 0,30-0,40 $\mathrm{m}$ e separadas em agregados maiores e menores que 0,25 mm. Foram realizadas análises químicas e físicas para caracterização do solo e determinados o carbono orgânico total, o carbono mineralizável, o carbono da biomassa microbiana, o carbono solúvel em água e a matéria orgânica lábil. Os resultados, indicaram que o plantio direto não foi capaz de manter os níveis de carbono nos agregados, quando comparado ao solo sob floresta nativa; a matéria orgânica desempenhou papel relevante na formação e estabilização de agregados maiores que $0,25 \mathrm{~mm}$; a porosidade, resistência ao penetrômetro e densidade do solo demonstraram que houve aumento da compactação do solo no sistema plantio direto, quando comparado ao solo sob floresta nativa, tendo a densidade se mostrado uma variável satisfatória para a avaliação de compactação no Latossolo Vermelho Eutroférrico; a matéria orgânica lábil apresentou potencial para ser utilizada em estudos de ciclagem de nutrientes e fenômenos de dispersão e floculação de argila.
\end{abstract}

Termos de indexação: cerrado; plantio direto; matéria orgânica do solo.

\footnotetext{
(1) Parte da Tese de Mestrado do primeiro autor, apresentada ao Departamento de Sol os da Universidade Federal de Viçosa - UFV. Recebido para publicação em julho de 2002 e aprovado em julho de 2003.

(2) Professor Adjunto, Departamento de Zootecnia da Universidade Católica de Goiás - UCG. Av. Universitária 1440, Caixa Postal 86, Setor Universitário, CEP 74605-010 Goiânia (GO). E-mail: mariaelo@terra.com.br

(3) Doutorando do Departamento de Solos, U niversidade Federal de Viçosa - UFV. Av. PH Rolfs s/n, Campus, CEP $36570-000$ Viçosa (MG). Bolsista do CNPq. E-mail: nelciols@yahoo.com.br

(4) Professor do Departamento de Solos, UFV. Bolsista do CNPq. E-mails: esm@ufv.br; liovando@solos.ufv.br

(5) Pesquisador da E mbrapa, Centro de Pesquisa Agropecuária dos Cerrados - CPAC. Caixa Postal 08223, CEP $73301-970$ Planaltina (DF). E-mail: jroberto@cpaac.embrapa.br
} 


\title{
SUMMARY: CARBON FORMS OF A TYPIC EUTROFERRIC RED LATOSSOL UNDER NO-TILLAGE IN A SAVANNA BIOGEOGRAPHIC SYSTEM
}

\begin{abstract}
The objective of this study was to evaluate physical and chemical soil properties and organic carbon dynamics in a Typic Eutroferric Red Latossol under different land uses (notill system, irrigated and non- irrigated crops, and native forest) in the savanna region of Santa Helena de Goiás, Goiás State, Brazil. Samples were collected from five soil layers (0.0-0.05, 0.05-0.10, 0.10-0.20, 0.20-0.30, and 0.30-0.40 m) and separated in aggregatesize groups of above or below $0.25 \mathrm{~mm}$. Chemical and physical analyses determined the total organic, mineralizable, microbial biomass, and water-soluble carbon, as well as thelabile organi c matter for soil characterization. Thefoll owing cond usions weredrawn: (a) carbon levels in the aggregates werelower in the no-till system compared to the soil under natural forest; (b) organic matter plays a relevant rolein thegenesis and stabilization of aggregates $>0.25 \mathrm{~mm}$; (c) compared to the soil under natural forest, an increased soil compaction was evidenced in the no-till system by theporosity, resistanceto penetrometer, and bulk density; (d) and soil density is a satisfactory variableto eval uatesoil compaction of theTypic E utrofic Red Latosol. Labileorganic matter has the potential to be used in studies of clay dispersion and flocculation phenomena besides nutrient cycling.
\end{abstract}

Index terms: savanna; no-till; soil organic matter.

\section{INTRODUÇÃO}

As investigações sobre o cerrado, considerando seus aspectos bióticos e abióticos, revelam que este domínio deve ser entendido como um sistema biogeográfico, composto por ambientes diversificados entre si, pelo seu caráter fisionômico ou pela sua composição vegetal e animal. Tais ambientes constituem seis subsistemas interatuantes, que são o subsistema dos campos, do cerrado, do cerradão, das matas, das matas ciliares e o subsistema das veredas e dos ambientes al agadiços (Barbosa, 1996).

Quando se fal a em solos de cerrado, há quase uma sinonímia com Latossol os, possivel mente por serem os mais freqüentes e os mais utilizados (Ker et al., 1992). Estes, por sua vez, ocupam uma posição de relevo suave ondulado na paisagem, sendo mecanizáveis e com propriedades físicas favoráveis à agricultura (Resck et al., 1991). São solos profundos, bastante intemperizados e ricos em caulinita e óxidos de Fe eAl. Na maioria das vezes, apresentam acidez elevada e alta capacidade de fixação de P (Goedert, 1983).

Greenland \& Oades (1975) relataram que a estabilidade da estrutura do solo é resultante da presença de materiais orgâni cos einorgânicos, eArca \& Weed (1966) mostraram que os agentes que influem na formação e estabilização dos agregados do solo são: tamanho e distribuição das partículas elementares do solo; conteúdo de argila e tipo do argilomineral presente; natureza da saturação por cátions; presença esolubilidade dos sais em sol ução; fatores biológicos, incluindo produtos da decomposição bi ológica eorganismos vivos, eagentes cimentantes orgânicos. Por sua vez, Tisdall \& Oades (1982) evidenciaram a correl ação entre o conteúdo de C orgânico e a estabilidade dos agregados em água.

Quando ocorre a substituição de ecossistemas naturais por agroecossistemas com culturas, percebe-se o declínio no conteúdo de $\mathrm{C}$ do solo, decorrente da redução no aporte e do aumento da decomposição da matéria orgânica. Apesar de a matéria orgânica do solo aumentar sob o sistema plantio direto e apresentar el evado aporte de resíduos, deve-se considerar que este aumento também depende de outros fatores, tais como: clima, principalmente temperatura, precipitação, textura e mineralogia do solo (Alvarez \& Lavado, 1998). O aumento no conteúdo de $C$ pode ser limitado em sol os de textura fina e mal drenados e em locais onde baixas temperaturas dificultem a decomposição da matéria orgânica (Angers et al., 1997; Paustian et al., 1997).

Os sistemas de manejo necessitam então ser investigados regi onal mente sob diferentes condições de clima e solo, otimizando o conhecimento sobre o potencial do manejo conservacionista e os efeitos na mudança global.

O plantio direto caracteriza-se pela semeadura realizada diretamente sobre os restos culturais do cultivo anterior, sem nenhum preparo do sol o (aração e gradagem). A permanência dos resíduos vegetais na superfície protege o solo contra a erosão. A cobertura morta, formada pel o acúmul o de resíduos vegetais na camada superficial, dimi nui as oscilações de temperatura e umidade na superfície e contribui para a manutenção de temperaturas mais amenas e maior retenção de água no solo em períodos 
quentes e de estiagem prol ongada (Colozzi-Filho et al., 1999). Essas características do plantio direto determinam profundas al terações nas propriedades químicas, físicas e biológicas do solo que, por sua vez, refletem-se na fertilidade e na eficiência do uso de nutrientes pelas culturas (Muzilli, 1983; Sidiras \& Pavan, 1985; Sá, 1993, 1999).

Para um melhor entendimento, a matéria orgânica do sol o pode ser dividida em compartimentos lábeis e estáveis (Theng et al., 1989). Assim, a camada de manta orgânica, as raízes de plantas, macrorganismos, microrganismos, fração leve, formas solúveis em água esubstâncias não-húmi cas podem ser incluídos no compartimento lábil. As formas lábeis de $\mathrm{C}$ têm grande importância nos processos de formação e estabi lização de agregados, muito embora os estudos se concentrem mais nas substâncias húmicas e suas relações com o material mineral.

U ma das formas de quantificar o compartimento lábil é a determinação do $\mathrm{C}$ mineralizável, que se origina da quebra das moléculas orgânicas pela degradação dos substratos incorporados ao solo, convertido em energia e biomassa pelo processo da respiração.

Outra forma é a determinação da biomassa microbiana, que pode ser definida como a parte viva da matéria orgânica do solo, excluindo-se as raízes de plantas e os grandes animais (Siqueira, 1993), e que representa de 1 a $4 \%$ do carbono total do solo. É importante sob três aspectos: (a) por ser formada, em parte, por células vegetativas em plena atividade funcional, écapaz de promover alterações importantes no sistema solo, atuando como um catalisador para as transformações da matéria orgânica do solo; (b) por apresentar grandequantidade e por ser o maior componentelábil da matéria orgânica, torna-se um importante reservatório de nutrientes potencialmente disponíveis para as plantas, e (c) por representar um indicador de grande sensibilidade para avaliar as mudanças no solo, sendo influenciada pelas adubações, pelos métodos de cultivo e pelas condições edafocl imáticas.

A forma de $\mathrm{C}$ associada à superfície de troca dos solos e extraída por bissulfato de $\mathrm{Na}$ e o $\mathrm{C}$ solúvel em água, associado às formas orgânicas derivadas de oligossacarídeos e compostos derivados de ligninas (Guggenberger \& Zech, 1994), também estão associados ao compartimento lábil deC. O C solúvel é um constituinte comum do solo e da água superficial. Estas substâncias solúveis, presentes na água contida nos poros do solo, podem exercer substancial influência sobre os processos iônicos, ciclo de nutrientes, dinâmica da comunidade microbiana eintemperismo mineral nos sol os (Evans J r. et al., 1988).

Assim, o presente trabal ho teve como objetivo estudar comodiferentes formas deC são influenciadas pelo sistema plantio direto, irrigado e de sequeiro, na região dos Cerrados.

\section{MATERIAIS E MÉTODOS}

\section{Caracterização da área}

O estudo foi realizado no município de Santa Helena de Goiás, em Goiás, área representativa na região sudoeste do estado. O clima se enquadra no tipo Aw de Köppen. O relevo varia de suavemente ondulado a plano, com altitude de $560 \mathrm{~m}$, e a vegetação predominante é a floresta nativa, Cerradão. Como material de origem há predomínio de basalto, eo sol o seapresenta sem erosão evidente, com estrutura forte muito pequena granular, muito poroso, consistência macio friável, plástico e pegajoso, excessivamente drenado, sendo classificado como Latossolo Vermel ho Eutroférrico típico textura argilosa (EMBRAPA, 1999).

\section{Coleta do material de solo}

As amostras de solo foram col etadas em agosto de 1995 em áreas sob o sistema plantio direto irrigado (PI), plantio direto de sequeiro (PS) e floresta nativa (FN), no terço médio das encostas. Estes sítios de tratamentos eram áreas adjacentes, distando $100 \mathrm{~m}$. Nos últimos anos, a área foi utilizada no plantio de culturas de acordo com o quadro 1.

Para cada sistema de manejo (PI, PS eF N), foram avaliados três perfis com distância em torno de 100 m entre cada um, onde foram retiradas amostras nas profundidades de: $0,00-0,05,0,05-0,10,0,10-0,20$, $0,20-0,30$ e 0,30-0,40 m. Para cada profundidade e cada perfil, coletaram-se três amostras indeformadas, com o auxílio de anel volumétrico, para análises de densidade do solo. Para determinação da umi dade, as amostras de solo, após coletadas, foram acondicionadas em recipientes

\section{Quadro 1. Cultivos na área em estudo entre os anos} de 1990 a 1995

\begin{tabular}{lll}
\hline & \multicolumn{2}{c}{ Plantio direto } \\
\cline { 2 - 3 } & Irrigado & Sequeiro \\
\hline 90/91 - safra & Milho & Milho \\
90/91 - inverno & Feijão & \\
91/92 - safra & Milho & Soja \\
91/92 - inverno & Feijão & \\
92/93 - safra & Milho & Milho \\
92/93 - inverno & Milheto & \\
93/94 - safra & Soja & Soja \\
93/94 - safrinha & Feijão & Milheto \\
94/95 - safra & Milho & Soja \\
94/95 - safrinha & Feijão & Milheto \\
94/95 - inverno & Crotalária & \\
\hline
\end{tabular}

Fonte: Sementes Fartura (informação pessoal). 
próprios, imediata e hermeticamente fechados. As amostras foram secas à sombra e passadas por peneiras de $4 \mathrm{~mm}$ demalha. Posteriormente, com o emprego de agitador e durante $15 \mathrm{~min}$ a 60 ciclos por segundo, os solos foram separados em duas classes de agregados: maiores que $0,25 \mathrm{~mm}$ e menores que $0,25 \mathrm{~mm}$.

\section{Análises físicas}

A análise textural foi realizada pelo método da pi peta para silte eargila, tendo sidoa areia separada por peneiramento (EMBRAPA, 1997); a densidade do solo, pelo método do anel volumétrico (EMBRAPA, 1997); a densidade de partículas, pelo método do balão volumétrico, utilizando-se álcool etílico como líquido penetrante (EMBRAPA, 1997), e a percentagem de agregados, por via seca em agitador mecânico (EMBRAPA, 1997).

Foram também determinadas: a umidade residual, após as amostras de solo permanecerem em estufa a $105-110{ }^{\circ} \mathrm{C}$ por $24 \mathrm{~h}$ (EMBRAPA, 1997); a porosidade total, a partir dos val ores de densidade do solo e densidade de partículas (EMBRAPA, 1997), e a argila dispersa em água, de acordo com J ucksch (1987). Tal método utiliza um agitador horizontal com 200 oscilações por minuto, em que 30 g de terra fina seca ao ar mais $100 \mathrm{~mL}$ de água dei onizada são acondicionados em frasco de vidro de $200 \mathrm{~mL}$, vedado com rolha, e agitados durante três horas. Em seguida, o material foi transferido para uma proveta de $500 \mathrm{~mL}$, passando por peneira de $0,21 \mathrm{~mm}$ de malha. Completou-seovolume da proveta com água destilada e determinou-sea argila dispersa em água (ADA), pelo método da pipeta, de acordo com adaptação do método proposto por E mbrapa (1997).

A resistência ao penetrômetro foi realizada com penetrômetro demão, efoi feita segundoa orientação do fabricante (Wykehan Farrance Engineering Limited).

\section{Análises químicas}

Cátions trocáveis - o cál cio e o magnésio trocáveis foram extraídos com $\mathrm{KCl} 1 \mathrm{~mol} \mathrm{~L}^{-1}$ e determinados por espectrofotometria de absorção atômica. O K extraído pelo Mehlich-1 foi determinado por fotometria de emissão de chama; acidez trocável extração com KCl $1 \mathrm{~mol} \mathrm{~L}^{-1}$ e determinação por titulometria; fósforo - foi extraído pelo Mehlich-1 e determinado por col orimetria; carbono orgâni co total - utilizado o processo de Walkley - Black. Todas as análises químicas foram executadas e cal culadas de acordo com Defelipo \& Ribeiro (1981).

\section{Formas de carbono}

A matéria orgânica lábil foi determinada de acordo com Medeir ros \& Mendonça (1994), sendo o C orgânico dosado, conforme Bartlett \& Ross (1988). Para determinar o carbono solúvel em água (CSA), utilizou-se uma amostra de $10 \mathrm{~g}$ de solo de cada tratamento em $20 \mathrm{~mL}$ de $\mathrm{H}_{2} \mathrm{O}$, sendo a suspensão agitada por $15 \mathrm{~min}$, centrifugada e, posteriormente, filtrada em papel umedecido com $\mathrm{H}_{2} \mathrm{O}$. A determinação do carbono foi feita por colorimetria (Bartlett \& Ross, 1988). O carbono mineralizável foi quantificadoa partir da evolução de $\mathrm{CO}_{2}$ (Zibilske, 1994), com incubação de 23 dias. A biomassa microbiana de $C$ foi determinada, segundo o método proposto por Brookes et al. (1985), com algumas adaptações do método de extração de GamaRodrigues et al. (1994).

\section{Análise estatística}

Os dados foram submetidos à análise de variância, no delineamento de blocos ao acaso, conforme o seguinte modelo matemático:

$$
\begin{aligned}
& \hat{y} \mathrm{ijk}=\mu+\alpha i+\beta j+(\alpha \beta) i j+\delta k+(\alpha \delta) i k+(\beta \delta) j k+\varepsilon \\
& \text { sendoi }=1,2,3 ; \quad j=1,2,3 ; \quad k=1,2,3,4,5
\end{aligned}
$$

em que: $\hat{y}$ ijk =observação referenteao i-ésimo bl oco (repetiçẫo) no j-ésimo tipo de manejo e k-ésima camada (profundidade); $\mu=$ média geral; $\alpha \mathrm{i}=$ efeito do i-ési mo bloco; $\beta \mathrm{j}=$ =efeito doj-ésimo tipo de manejo; $(\alpha \beta) i j=$ efeito da interação entre o i-ésimo bloco e o j-ésimotipo de manejo (erroa); $\delta k$ =efeito da k-ésima camada (profundidade); $(\alpha \delta) i k=$ efeito da interação entre o i-ésimo bloco e a k-ésima camada (profundidade) (erro b); ( $\beta \delta) j k$ = efeito da interação entre o j-ésimo tipo de manejo e a k-ésima camada (profundidade), e $\varepsilon=$ erro experimental. Para cada camada, os graus de liberdade referentes aos tipos de manejo foram desdobrados nos seguintes contrastes ortogonais:

$$
\begin{array}{ll}
\mathrm{C} 1=\mathrm{PI}+\mathrm{PS}-2 \mathrm{FN} & \text { i-ésimo } \\
\mathrm{C} 2=\mathrm{PI}-\mathrm{PS} & \text { j-ésimo }
\end{array}
$$

\section{RESULTADOS E DISCUSSÃO}

\section{Características químicas e físicas do solo}

Os valores negativos de $\Delta \mathrm{pH}$ para todos os tratamentos demonstraram o predomínio de cargas negativas no solo, tendo o solo sob floresta nativa apresentado mais cargas líquidas, condição esta que favorece a dispersão de argilas, sobretudo em profundidades maiores (Quadro 2).

Comparando o plantio direto irrigado (PI) e o de sequeiro (PS), observaram-se, em todas as profundidades, valores absol utos mai ores para o PS, o que o predispõeà maior dispersão de argila queo PI .

Os teores de $\mathrm{P}$ diminuíram em profundidade, especialmente no sol o sob floresta nativa (FN). Para oPI ePS, houve aumento na camada de 0,05-0,10 m, decorrente da adubação com NPK utilizada no cultivo. Para oCa e Mg, e mesmo para oK, também reposto por meio de adubações periódicas, essa 
tendência não ocorreu. Os altos valores de K, para o solo sob floresta nativa ( $F N)$, estão relacionados com a proximidade do embasamento cristalino, o que leva a supor que este material tenha intemperismo e pedogênese no local e pode estar influenciando as difer enças observadas entre as situações estudadas.

Além disso, durante a coleta das amostras, observou-sea presença de mica ao longo do perfil do sol o sob floresta nativa. Essa diferença no material de origem não deve acarretar alteração em outras propriedades do solo. O plantio irrigado e o de sequeiro não diferiram entre si quanto aos valores $\mathrm{deH}+\mathrm{Al}$ até 0,20 m deprofundidade, apresentandose, no sol o sob floresta nativa, superiores aos demais.

O conteúdo de argila foi maior no $\mathrm{PI}$ até à profundidade de $0,3 \mathrm{~m}$, embora numericamente este comportamento tenha-se repetido em todas as profundidades estudadas (Quadro 3). Tal fato pode ter ocorrido em razão da maior quebra dos agregados sujeitos aos ciclos de umedecimento e secagem, da maior movimentação de máquinas e equipamentos agrícolas, decorrente da irrigação, e das maiores safras anuais.
Observando os valores de argila dispersa em água (Quadro 4), quando se contrastou o PD com a FN, verificou-se que eles foram maiores para a floresta nativa, nas profundidades compreendidas entre $0,05-0,40 \mathrm{~m}$. Pode-se dizer que esse seja um comportamento natural, associado à carga líquida do solo $(\Delta \mathrm{pH})$. Os val ores de densidade de partícula (Dp) evidenciam altos teores de óxidos de Fe, que, por apresentarem maiores densidades, el evam esses valores até $2,97 \mathrm{~g} \mathrm{~cm}^{-3}$, nos sistemas avaliados. O contraste significativo entre PD eF $\mathrm{N}$ até $0,10 \mathrm{~m}$ de profundidade(Quadro 3) evidencia a importância da matéria orgânica em diminuir a densidade de partícula.

Analisando o contraste entre plantio direto irrigado (PI) e de sequeiro (PS), verificou-se que a ADA não diferiu até os $0,20 \mathrm{~m}$ de profundidade, tendo os maiores valores sido encontrados no solo sob sequeiro, nas camadas de 0,20-0,30 m e de 0,30$0,40 \mathrm{~m}$. Para a $\mathrm{Dp}$, a diferença não foi significativa até $0,20 \mathrm{~m}$, apresentando-se maior no plantio direto de sequeiro, nas profundidade de 0,20-0,30 me0,300,40 m (Quadro 4).

Quadro 2. Valores de pH em água, $\mathrm{pH}$ em $\mathrm{KCl}$ e DpH, teores de $\mathrm{P}, \mathrm{K}, \mathrm{Ca}, \mathrm{Mg}, \mathrm{H}+\mathrm{Al}$, soma de bases (SB), CTC efetiva (CTCe), CTC total (CTC), alumínio e V, de um Latossolo Vermelho Eutroférrico Típico sob plantio direto irrigado (PI), plantio direto de sequeiro (PS) e floresta nativa (FN), e valores de contrastes para as mesmas variáveis

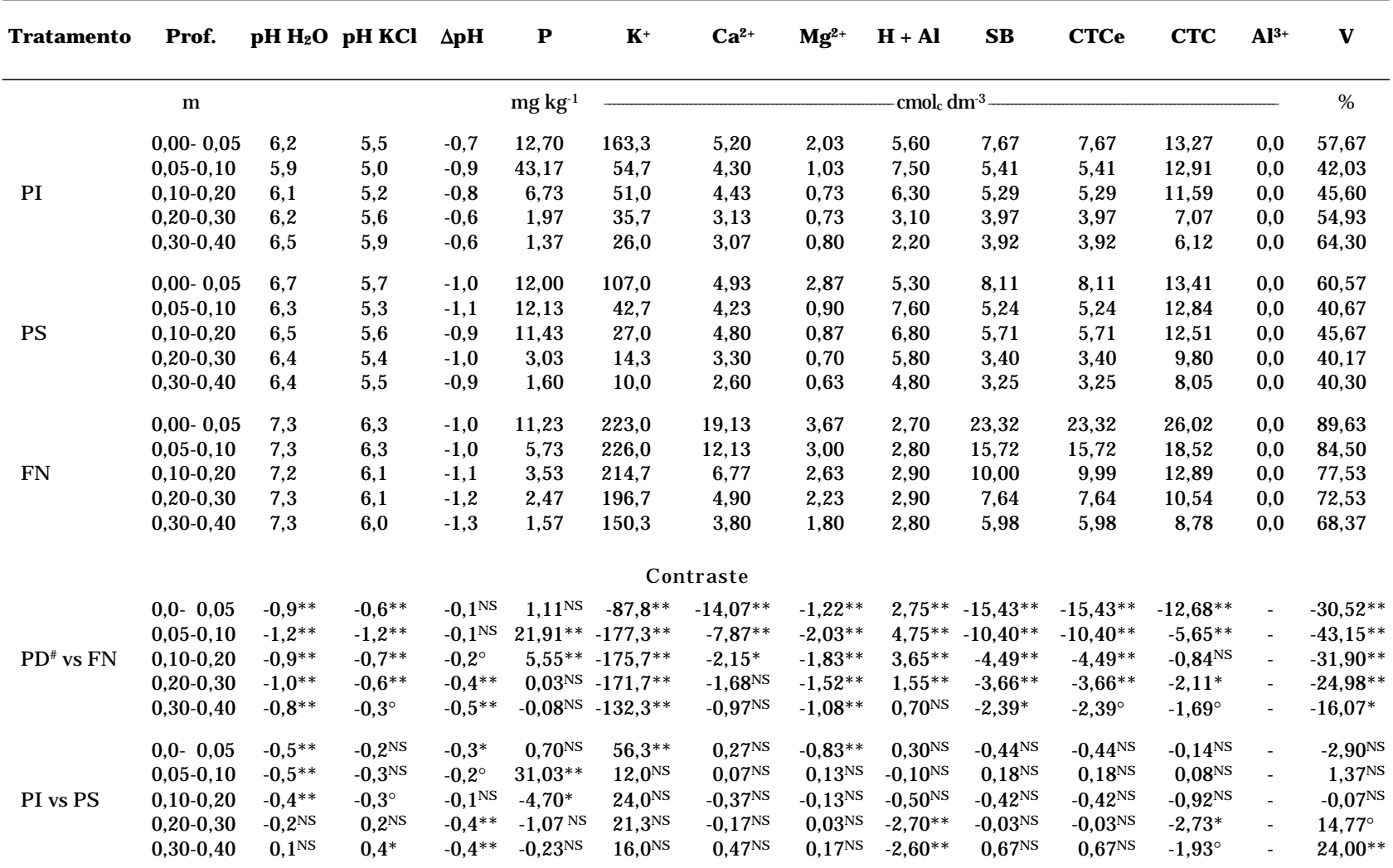

$\mathrm{NS}^{\circ}{ }^{*}$ * e** : não-significativo e significativo a 10,5 e $1 \%$, respectivamente. ${ }^{\#}=$ Plantio direto irrigado e de sequeiro. 
Quadro 3. Valores de areia grossa (AG), areia fina (AF), silte (SIL), argila (ARG) e umidade (UM) de um Latossolo Vermelho Eutroférrico Típico sob plantio direto irrigado (PI), plantio direto de sequeiro (PS) e floresta nativa (FN), em cinco profundidades, evalores de contrastes para as mesmas variáveis

\begin{tabular}{|c|c|c|c|c|c|c|}
\hline Tratamento & Profundidade & Areia grossa & Areia fina & Silte & Argila & Umidade \\
\hline \multicolumn{7}{|c|}{ _dag $\mathrm{kg}^{-1}$} \\
\hline $\mathrm{PI}$ & $\begin{array}{l}0,0-0,05 \\
0,05-0,10 \\
0,10-0,20 \\
0,20-0,30 \\
0,30-0,40\end{array}$ & $\begin{array}{l}15 \\
14 \\
14 \\
14 \\
14\end{array}$ & $\begin{array}{l}13 \\
13 \\
13 \\
13 \\
13\end{array}$ & $\begin{array}{l}21 \\
19 \\
18 \\
19 \\
19\end{array}$ & $\begin{array}{l}51 \\
54 \\
55 \\
54 \\
54\end{array}$ & $\begin{array}{l}35 \\
33 \\
33 \\
31 \\
30\end{array}$ \\
\hline PS & $\begin{array}{l}0,0-0,05 \\
0,05-0,10 \\
0,10-0,20 \\
0,20-0,30 \\
0,30-0,40\end{array}$ & $\begin{array}{l}17 \\
18 \\
17 \\
17 \\
16\end{array}$ & $\begin{array}{l}16 \\
17 \\
17 \\
17 \\
16\end{array}$ & $\begin{array}{l}25 \\
20 \\
22 \\
19 \\
18\end{array}$ & $\begin{array}{l}42 \\
45 \\
44 \\
47 \\
50\end{array}$ & $\begin{array}{l}14 \\
20 \\
22 \\
25 \\
24\end{array}$ \\
\hline $\mathrm{FN}$ & $\begin{array}{l}0,0-0,05 \\
0,05-0,10 \\
0,10-0,20 \\
0,20-0,30 \\
0,30-0,40\end{array}$ & $\begin{array}{l}12 \\
11 \\
10 \\
11 \\
11\end{array}$ & $\begin{array}{l}13 \\
15 \\
14 \\
15 \\
15\end{array}$ & $\begin{array}{l}27 \\
23 \\
20 \\
24 \\
19\end{array}$ & $\begin{array}{l}48 \\
51 \\
56 \\
50 \\
55\end{array}$ & $\begin{array}{l}28 \\
26 \\
25 \\
24 \\
23\end{array}$ \\
\hline \multicolumn{7}{|c|}{ Contraste } \\
\hline PD\# vs FN & $\begin{array}{l}0,0-0,05 \\
0,05-0,10 \\
0,10-0,20 \\
0,20-0,30 \\
0,30-0,40\end{array}$ & $\begin{array}{l}4,3^{* * *} \\
4,7^{* * *} \\
5,8^{* * *} \\
4,8^{* * *} \\
4,3^{* * *}\end{array}$ & $\begin{array}{r}1,2^{\circ} \\
0,5^{\mathrm{NS}} \\
0,8^{\mathrm{NS}} \\
-0,3^{\mathrm{NS}} \\
-0,7^{\mathrm{NS}}\end{array}$ & $\begin{array}{l}-4,2^{*} \\
-3,5^{\circ} \\
-0,2^{\text {NS }} \\
-5,8^{* *} \\
-0,5^{\text {NS }}\end{array}$ & $\begin{array}{c}-1,5^{\mathrm{NS}} \\
-1,3^{\mathrm{NS}} \\
-6,5^{* *} \\
0,7^{\mathrm{NS}} \\
-3,0^{\mathrm{NS}}\end{array}$ & $\begin{array}{l}- \\
- \\
- \\
- \\
-\end{array}$ \\
\hline PI vs PS & $\begin{array}{l}0,0-0,05 \\
0,05-0,10 \\
0,10-0,20 \\
0,20-0,30 \\
0,30-0,40\end{array}$ & $\begin{array}{l}-2,0^{*} \\
-4,7^{* * *} \\
-3,0^{* *} \\
-3,0^{* *} \\
-1,3^{\text {NS }}\end{array}$ & $\begin{array}{l}-3,0^{* *} \\
-3,7^{* * *} \\
-4,3^{* * *} \\
-4,7^{* * *} \\
-2,7^{* *}\end{array}$ & $\begin{array}{l}-4,3^{\circ} \\
-0,3^{\mathrm{NS}} \\
-3,7^{\circ} \\
0,3^{\mathrm{NS}} \\
1,0^{\mathrm{NS}}\end{array}$ & $\begin{array}{c}9,7^{* *} \\
8,0^{* *} \\
11,0^{* * *} \\
7,3^{*} \\
3,3^{\text {NS }}\end{array}$ & $\begin{array}{l}- \\
- \\
- \\
- \\
-\end{array}$ \\
\hline
\end{tabular}

NS,,$*$, ** e ***: não-significativo e significativo a 10, 5, 1 e 0,1 \%. * $=$ plantio direto sob irrigação e de sequeiro.

A resistência do solo ao penetrômetro (RP) mostrou diferença a partir de 0,05 m de profundidade, tendo apresentado valores menores no solo sob floresta nativa, comparados aos do PD (Quadro 4). Apesar de ser uma medida simples, ela parece representar muito bem o que acontece no solo, onde a resistência à penetração aumenta de acordo com a maior interferência antrópica. Observaram-se maiores val ores de RP nas camadas de 0,10-0,20 m, o que caracteriza adensamento ou compactação subsuperficial.

Mesmo sendo de ocorrência natural nos Latossolos Vermel hos Eutroférricos da região, quando se compararam as áreas cultivadas sob plantio direto com o solo sob floresta nativa, essa diferença foi incrementada. Conseqüentemente, a densidade do sol o (Ds) foi maior em PD, comparada à da floresta nativa, em todas as profundidades estudadas (Quadro 4). Para o contrasteentreplantio direto irrigado e de sequeiro (Quadro 4), a RP foi numericamentemaior no plantio dir eto de sequeiro, em todas as profundidades, e estatisticamentemaior nas camadas de 0,05-0,10, 0,10-0,20, 0,20-0,30 e 0,30$0,40 \mathrm{~m}$. Tal fato, natural mente, aconteceu por causa dos mai ores teores de umi dadeadvindos da irrigação no PI, comparados aos do PS (Quadro 2).

Na realidade, como a RP não foi ajustada para a umidade do solo, esta se prestou somente como uma medida aproximada da compactação do solo. Mesmo assim, pode-se inferir que o cultivo sob plantio direto levou ao aumento da resistência à penetração, quando comparado com o solo sob floresta nativa, uma vez queo PI estava com umidade superior à da FN, quando da amostragem (Quadro 3).

Constatou-se que os maiores valores de RP ocorreram nas camadas compreendidas entre 0,05$0,30 \mathrm{~m}$ de profundidade do PS, pois a amostragem foi realizada no mês de agosto, ou seja, no período seco do ano. Neste mesmo período, a área sob PI esteve sendo cultivada e recebendo irrigação, conseqüentemente, apresentando menor RP, por causa do maior teor de umidade do sol o (Quadro 4). 
Quadro 4. Argila dispersa em água (ADA), densidade de partícula (Dp), porosidade, resistência ao penetrômetro (RP) e densidade do solo (Ds) de um Latossolo Vermelho E utroférrico Tí́pico sob plantio direto irrigado (PI), plantio direto de sequei ro (PS) e floresta nativa (F N), em cinco profundidades, e valores de contrastes para as mesmas variáveis

\begin{tabular}{|c|c|c|c|c|c|c|}
\hline Tratamento & Profundidade & ADA & Porosidade & Dp & Ds & $\mathbf{R} \mathbf{P}$ \\
\hline & $\mathrm{m}$ & dag kg-1 & $m^{3} m^{-3}$ & $\mathrm{~kg} \mathrm{dm}^{-3}$ & $\mathrm{~g} \mathrm{~cm}^{-3}$ & $\mathrm{~kg} \mathrm{~cm}^{2}$ \\
\hline $\mathrm{PI}$ & $\begin{array}{l}0,00-0,05 \\
0,05-0,10 \\
0,10-0,20 \\
0,20-0,30 \\
0,30-0,40\end{array}$ & $\begin{array}{r}8 \\
7 \\
10 \\
7 \\
2\end{array}$ & $\begin{array}{l}0,647 \\
0,603 \\
0,584 \\
0,615 \\
0,651\end{array}$ & $\begin{array}{l}2,81 \\
2,88 \\
2,82 \\
2,95 \\
2,97\end{array}$ & $\begin{array}{l}0,99 \\
1,14 \\
1,17 \\
1,14 \\
1,04\end{array}$ & $\begin{array}{r}1,10 \\
8,00 \\
16,67 \\
13,33 \\
10,00\end{array}$ \\
\hline PS & $\begin{array}{l}0,00-0,05 \\
0,05-0,10 \\
0,10-0,20 \\
0,20-0,30 \\
0,30-0,40\end{array}$ & $\begin{array}{r}6 \\
6 \\
7 \\
10 \\
10\end{array}$ & $\begin{array}{l}0,648 \\
0,630 \\
0,626 \\
0,635 \\
0,656\end{array}$ & $\begin{array}{l}2,85 \\
2,83 \\
2,85 \\
2,88 \\
2,91\end{array}$ & $\begin{array}{l}1,00 \\
1,05 \\
1,07 \\
1,05 \\
1,00\end{array}$ & $\begin{array}{r}1,57 \\
18,33 \\
24,67 \\
22,00 \\
16,33\end{array}$ \\
\hline FN & $\begin{array}{l}0,00-0,05 \\
0,05-0,10 \\
0,10-0,20 \\
0,20-0,30 \\
0,30-0,40\end{array}$ & $\begin{array}{r}7 \\
10 \\
11 \\
13 \\
15\end{array}$ & $\begin{array}{l}0,703 \\
0,668 \\
0,656 \\
0,681 \\
0,670\end{array}$ & $\begin{array}{l}2,63 \\
2,80 \\
2,89 \\
2,94 \\
2,97\end{array}$ & $\begin{array}{l}0,78 \\
0,93 \\
1,00 \\
0,94 \\
0,98\end{array}$ & $\begin{array}{l}0,47 \\
4,67 \\
9,00 \\
7,33 \\
6,67\end{array}$ \\
\hline \multicolumn{7}{|c|}{ Contraste } \\
\hline$P D \#$ vs FN & $\begin{array}{l}0,00-0,05 \\
0,05-0,10 \\
0,10-0,20 \\
0,20-0,30 \\
0,30-0,40\end{array}$ & $\begin{array}{l}-0,2^{\mathrm{NS}} \\
-3,0^{\circ} \\
-2,6^{\circ} \\
-4,6^{* *} \\
-8,7^{* * *}\end{array}$ & $\begin{array}{l}-0,55^{* * *} \\
-0,51^{* * *} \\
-0,51^{* * *} \\
-0,56^{* * *} \\
-0,17^{*}\end{array}$ & $\begin{array}{l}0,20^{* * *} \\
0,05^{*} \\
-0,05^{*} \\
-0,03^{\text {NS }} \\
-0,03^{\text {NS }}\end{array}$ & $\begin{array}{l}0,21^{* * *} \\
0,16^{* * *} \\
0,12^{* * *} \\
0,15^{* * *} \\
0,04^{*}\end{array}$ & $\begin{array}{r}0,87^{N S} \\
8,50^{* * *} \\
11,67^{* * *} \\
10,33^{* * *} \\
6,50^{* *}\end{array}$ \\
\hline PI vs PS & $\begin{array}{l}0,00-0,05 \\
0,05-0,10 \\
0,10-0,20 \\
0,20-0,30\end{array}$ & $\begin{array}{l}1,7^{\mathrm{NS}} \\
2,4^{\mathrm{NS}} \\
-3,4^{\circ} \\
-7,3^{* * *}\end{array}$ & $\begin{array}{l}-0,26^{* *} \\
-0,42^{* * *} \\
-0,20^{*} \\
-0,04^{\mathrm{NS}}\end{array}$ & $\begin{array}{c}0,05^{N S} \\
-0,03^{N S} \\
0,08^{*} \\
0,06^{*}\end{array}$ & $\begin{array}{l}0,09^{* *} \\
0,11^{* * *} \\
0,09^{* *} \\
0,03^{\mathrm{NS}}\end{array}$ & $\begin{array}{c}-10,33^{* * *} \\
-8,00^{* *} \\
-8,67^{* *} \\
-6,33^{*}\end{array}$ \\
\hline
\end{tabular}

$\overline{\mathrm{NS}},{ }^{\circ}, *, * *$ e**: não-significativo e significativo a $10,5,1$ e 0,1. ${ }^{*}=$ plantio direto sob irrigação e de sequeiro.

Dessa forma, os dados de PORO, RP e Ds demonstram que houve aumento da compactação do sol o no sistema plantio direto, quando comparados aos do sol o sob floresta nativa.

\section{Características do material orgânico do solo}

\section{Formas de carbono nos agregados maiores e menores que 0,25 $\mathrm{mm}$}

De maneira geral, para todas as formas de C analisadas, foram obtidos maiores valores para os agregados menores que $0,25 \mathrm{~mm}$, comparados aos dos agregados maiores que 0,25 mm (Figuras 1 a 5).

Anderson et al. (1981) encontraram resultados semelhantes, ao passo que Gupta \& Germida (1988) chegaram a conclusões opostas, quando estudaram algumas das formas de $\mathrm{C}$ em diferentes classes de agregados. Esses últimos encontraram maiores valores, da ordem de $13 \%$, quando compararam o conteúdo de $\mathrm{C}$ total acumulado em macroagregados triturados e não triturados.
A determinação do $C$ total se deu sem a trituração dos agregados, sendo possível que os val ores obtidos sejam relativos ao $\mathrm{C}$ contido na superfície dos agregados. Para os agregados menores que $0,25 \mathrm{~mm}$, que apresentam maior superfície de contato, é possível que sejam encontrados mai ores val ores deC.

Considerando o modelo de agregação proposto por Edwards \& Bremner (1967), em que os macroagre gados com al ta estabilidade e o diâmetro superior a $250 \mathrm{~mm}$ são constituídos por microagregados formados por partículas de argila, unidas à matéria orgânica por metais polivalentes e com diâmetro entre 2 e $250 \mathrm{~mm}$, é de se esperar que, após a trituração dos macroagregados, o C fisicamente protegi do possa ser mensurado, aumentando os seus valores em relação aos microagregados.

Pel o fato de, nos microagregados, a fração argila ser provavel mente mais expressiva, pode-se inferir que há uma maior interação desta com as frações orgânicas do solo. Além disso, espera-se que as formas de $C$ para interagir com as partículas 


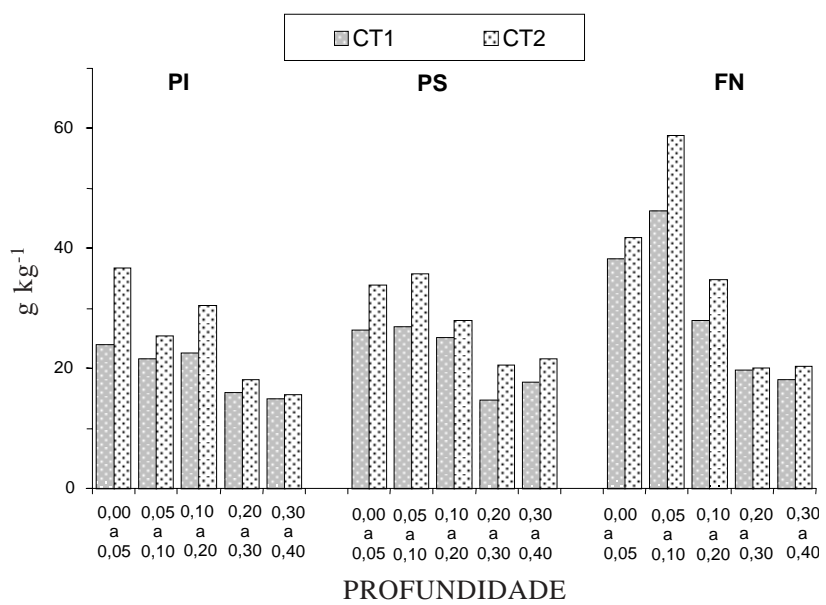

Figura 1. Carbono orgânico total nos agregados maiores e menores que $0,25 \mathrm{~mm}$, respectivamente (CT1 e CT2), de solos sob plantio direto irrigado (PI), plantio di reto de sequeiro (PS) e floresta nativa (FN), em cinco profundidades.

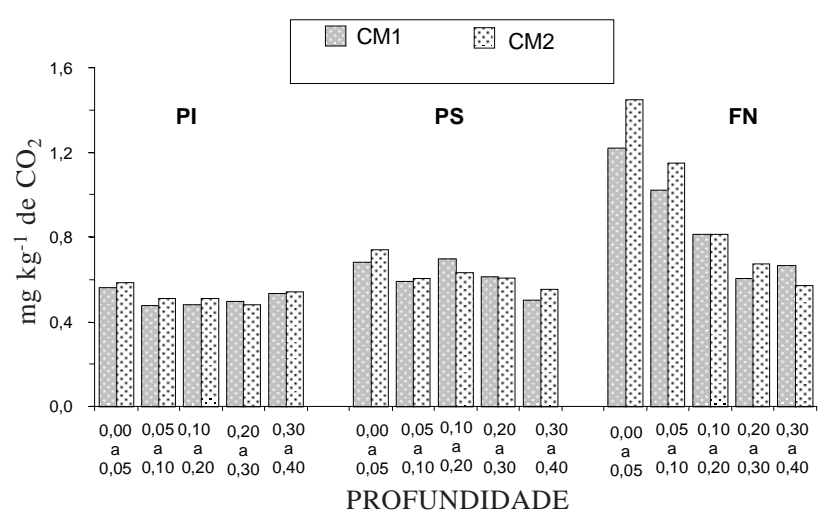

Figura 2. Carbono mineralizável nos agregados maiores e menores que $0,25 \mathrm{~mm}$, respectivamente (CM1 e CM2), de solos sob plantio direto irrigado (PI), plantio direto de sequeiro (PS) e floresta nativa (FN), em cinco profundidades.

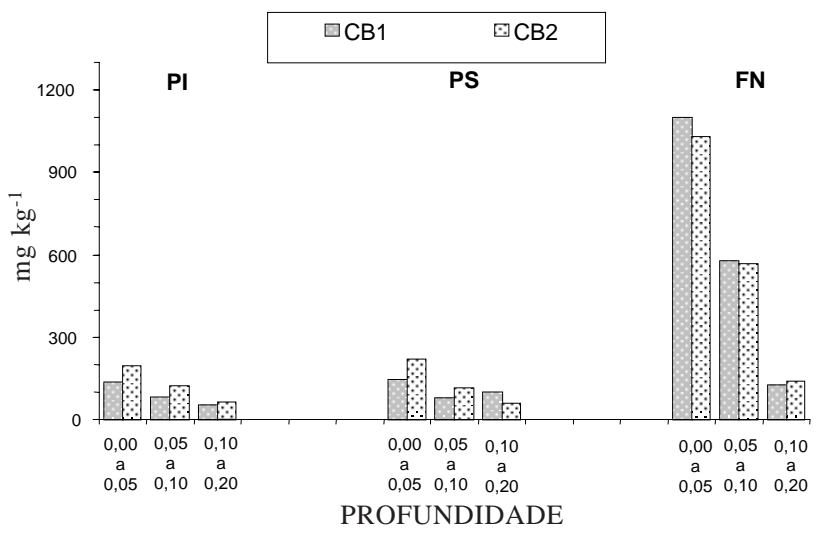

Figura 3. Carbono da biomassa microbiana nos agregados maiores e menores que $0,25 \mathrm{~mm}$, respectivamente (CB1 e CB2), de solos sob plantio direto irrigado (PI), plantio direto de sequeiro (PS) e floresta nativa (FN), em três profundidades.

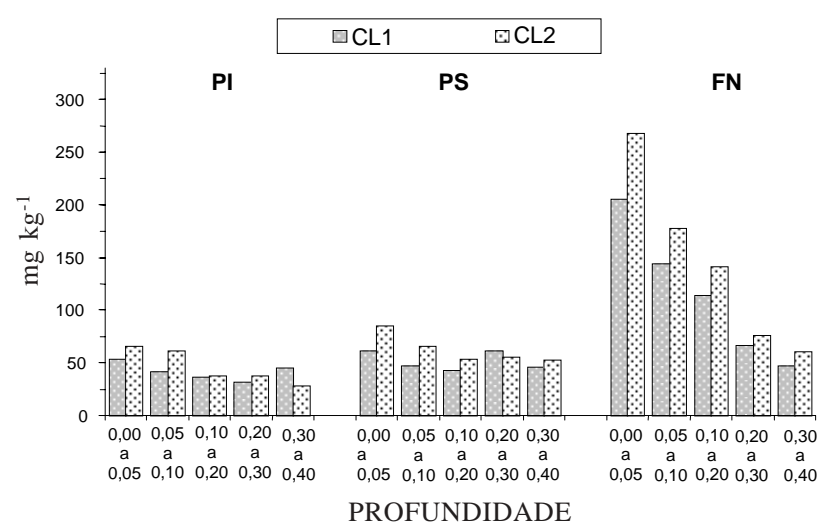

Figura 4. Carbono orgânico lábil nos agregados maiores e menores que $0,25 \mathrm{~mm}$, respectivamente (CL 1 e CL2), de solos sob plantio direto irrigado (PI), plantio direto de sequei ro (PS) e floresta nativa (FN), em cinco profundidades. menores estejam mais transformadas, enquanto, nos macroagregados, o material orgânico encontra-se menos alterado (Duxbury et al., 1989).

Os casos em que essa tendência não ocorreu foram pontuais, excetuando a forma de $\mathrm{C}$ solúvel em água (CS) para o sol o submetido ao plantio direto irrigado (PI), nas três profundidades compreendidas na faixa de 5-30 cm (Figura 5). O CS é uma forma lábil, facilmente perdida, e tem em sua constituição carboidratos, ácidos orgânicos de baixo peso molecular e uma pequena fração de ácidos fúlvicos (Stevenson, 1994).

No PI, como a entrada de material vegetal advindo da rotação de culturas e do cultivo mais intensivo durante o ano é maior $\left(\approx 14 \mathrm{Mg} \mathrm{ano}^{-1}\right.$ de matéria seca), pode-seinferir que este fator seja um dos responsáveis pelos valores superiores de CS encontrados. Além disso, a densidade do solo, nas camadas de 0,00-0,30 $\mathrm{m}$ de profundidade, também se apresenta com os maiores valores para o PI, caracterizando uma maior compactação nessa faixa.

Tal fato, somado às condições de umidade em virtude da irrigação, manteria esse material orgânico mais preservado, seja pela maior quantidade de água, seja pela menor capacidade de realizar trocas gasosas no sol o e pela dificuldade de movimentação da microbiota do solo sob plantio direto irrigado (PI).

Siqueira (1993) mostrou que a aeração do solo é crítica para a densidade populacional microbiana e para seus processos metaból icos equealterações na aeração são provocadas por vários mecanismos, como 


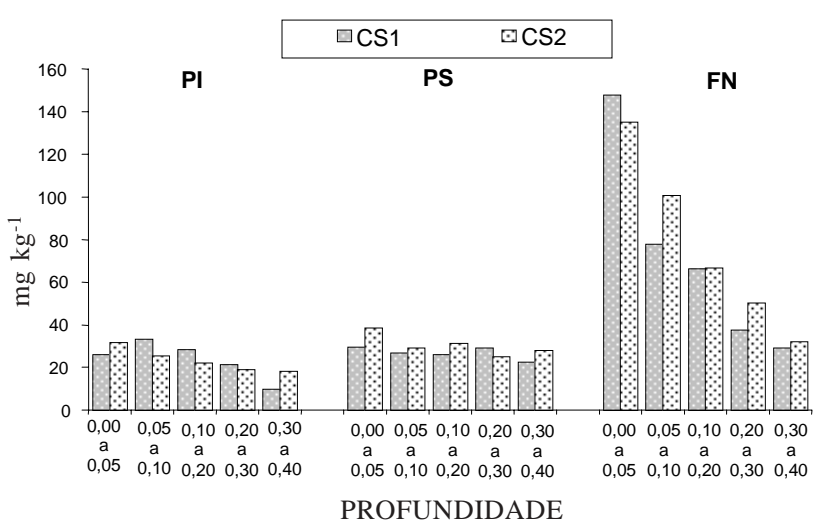

Figura 5. Carbono solúvel em água nos agregados maiores e menores que 0,25 $\mathrm{mm}$, respectivamente (CS1 e CS2), de solos sob plantio direto irrigado (PI), plantio direto sequeiro (PS) e floresta nativa (FN), em cinco profundidades.

compactação e inundação. A menor concentração de $\mathrm{O}_{2}$ modifica o potencial de oxirredução (Eh), a atividade e o metabolismo dos organismos do solo. Isso poderia justificar os maiores níveis deCS no PI e nas respectivas profundidades, uma vez que a transformação do C em formas mais estáveis estaria sendo prejudicada pelas condições ambientais.

Carbono orgânico total, carbono mineralizável e carbono da biomassa microbiana

Para o contraste entre o sol o sob plantio direto, irrigado e de sequeiro (PD), e o solo sob floresta nativa ( $\mathrm{FN})$, observou-se, de maneira geral, que as formas de C, sobretudo nas primeiras profundidades, foram maiores nesteúltimo (Quadro 5), mesmo para o C orgânico total (CT), caso em que foram obtidas as menores diferenças significativas entre os tratamentos. O sol o sob floresta nativa apresentou mai ores val ores apenas nas profundidades de 0,00$0,05 \mathrm{~m}$ e 0,05-0,10 m, para agregados maiores que $0,25 \mathrm{~mm}$, e de 0,05-0,10 m, para agregados menores que 0,25 mm (Quadro 5). Tal fato demonstra que, apesar do incremento do material orgânico no PD $\left(\approx 14 \mathrm{Mg} \mathrm{ha}^{-1} \mathrm{ano}^{-1}\right.$ de matéria seca, para o plantio direto irrigado, e 8,7 $\mathrm{Mg} \mathrm{ha}^{-1} \mathrm{ano}^{-1}$, para o plantio direto de sequeiro), os teores de CT não atingiram os níveis do sistema natural.

Para o C mineralizável (CM), observaram-se maiores valores para o solo sob floresta nativa, sobretudo até $0,30 \mathrm{~m}$ de profundidade, tanto para agregados maiores quanto para menores do que $0,25 \mathrm{~mm}$ (Quadro 5). As condições de temperatura interna, umidade e aeração, possibilitadas por menores oscilações destas variáveis no solo sob vegetação de mata, podem favorecer a população microbiana. A porosidadetotal mais el evada para o solo da floresta nativa (Quadro 4) confirma esse comportamento. Decorrente da menor cobertura vegetal, pode-se inferir que os solos sob PD estão sujeitos à maior oscilação na temperatura, quando comparados aos de floresta, e, portanto, toda a dinâmica de transformação pode ser alterada. Nessas condições, os sol os estariam também mais susceptíveis aos cicl os de umedecimento e secagem e às suas conseqüências.

Para o C da biomassa microbiana (CB), observousecomportamento semel hanteao das demais formas de C (Quadro 5), sendo significativas as diferenças até à profundidade de $0,10 \mathrm{~m}$ e onde o solo sob floresta nativa se apresentou numericamente maior em todas as profundidades.

Pode-se dizer que o solo da floresta nativa apresentou mai or população mi crobiana, o que pode ser justificado pela diversidade de espécies vegetais ali encontradas, refletindo diretamente numa maior amplitude de organismos vivos, que são favor ecidos pelas condições ambientais nesse solo. O CB representou de 1 a $4 \%$ do $C$ total do solo, podendo-se inferir que, no solo sob floresta nativa, as células vegetativas em plena atividade funcional são responsáveis por parte das diferenças físicas equímicas aí encontradas, uma vez quea biomassa microbiana funciona como um catalisador dessas alterações.

Quadro 5. Contrastes entre solos sob plantio di reto (PD) e sob floresta nativa (F N) para carbono orgânico total (CT), car bono mineralizável (CM) e carbono da biomassa mi crobiana (CB) em agregados maiores e menores que 0,25 mm, respectivamente (1 e 2), de um Latossolo Vermelho E utroférrico Típico, em cinco profundidades

\begin{tabular}{|c|c|c|c|c|c|c|c|}
\hline Tratamento & Profundidade & CT1 & CT2 & CM1 & CM2 & CB1 & CB2 \\
\hline & $\mathrm{m}$ & \multicolumn{2}{|c|}{$\mathrm{mg} \mathrm{kg}^{-1}$} & \multicolumn{2}{|c|}{$\ldots \mathrm{mg} \mathrm{kg}^{-1} \mathrm{de} \mathrm{CO}_{2}$} & \multicolumn{2}{|c|}{$\mathrm{g} \mathrm{kg}^{-1}$} \\
\hline & $\begin{array}{l}0,00-0,05 \\
0,05-0,10\end{array}$ & $\begin{array}{l}-13.090^{*} \\
-21.930^{* *}\end{array}$ & $\begin{array}{c}-6.615^{N S} \\
-28.140^{* *}\end{array}$ & $\begin{array}{l}-0,594 * * \\
-0,486 * *\end{array}$ & $\begin{array}{l}-0,790^{* *} \\
-0,596 * *\end{array}$ & $\begin{array}{l}-958,1^{* *} \\
-499,5^{*}\end{array}$ & $\begin{array}{l}-824,2^{*} \\
-447,9 *\end{array}$ \\
\hline$P D^{(1)}$ VS FN & $\begin{array}{l}0,10-0,20 \\
0,20-0,30 \\
0,00-0,05\end{array}$ & $\begin{array}{l}-4.110^{N S} \\
-4.350^{N S} \\
-1.800^{N S}\end{array}$ & $\begin{array}{r}-5.600^{N S} \\
-700^{N S} \\
-1.750^{N S}\end{array}$ & $\begin{array}{l}-0,223^{* *} \\
-0,047^{N S} \\
-0,151^{* *}\end{array}$ & $\begin{array}{l}-0,243^{* *} \\
-0,131^{\circ} \\
-0,023^{N S}\end{array}$ & $-49,0$ NS & 76,7 NS \\
\hline
\end{tabular}

$\mathrm{NS}^{\circ}{ }^{*}$ * $\mathrm{e}^{* *}$ : não-significativo e significativo a 10,5 e 1 \%, respectivamente. ${ }^{(1)}$ Plantio direto sob irrigação e de sequeiro. 
A anál ise química desse sol o apontou para teores de nutrientes no solo sob floresta nativa muito maiores queno PD que, mesmorecebendo adubações constantes, não foi capaz de manter os níveis tão el evados quanto na floresta (Quadro 2). Esses altos valores encontrados sãojustificados, em parte, pela el evada concentração de carbono na bi omassa, que, segundo Siqueira (1993), armazena val ores bastante el evados, como $100 \mathrm{~kg}$ de N, $80 \mathrm{~kg}$ de $\mathrm{P}, 70 \mathrm{~kg}$ de $\mathrm{K}$ e $11 \mathrm{~kg} \mathrm{ha}^{-1}$ de Ca.

Como a biomassa é reciclada cerca de dez vezes mais rapidamente que a fração orgânica morta do solo, grande parte dos nutrientes éliberada durante a sua reciclagem. Essa liberação ocorre de maneira gradual e de acordo com fatores que controlam a atividade e a população microbiana do solo.

Avaliando o contraste entre o solo sob plantio direto irrigado e o de sequeiro, verificou-se que não houve diferença tanto para agregados maiores quanto para menores que $0,25 \mathrm{~mm}$, quando se consi deraram as variáveis carbono orgânico total e carbono da biomassa microbiana (Quadro 6). Considerando que o teor de argila do PS foi inferior ao do PI e que é amplamente citada na literatura a associação de argila e carbono orgânico (Stevenson, 1994), eram esperados maiores valores de carbono no solo sob irrigação.

Embora estatisticamente não se verifiquem diferenças no conteúdo de $C$ total para o solo irrigado e de sequeiro, numericamente, pode-se observar que no PS foram encontrados os maiores valores de $C$ total (Figura 1). A irrigação, associada às condições de temperatura ambiente e do solo (por causa de sua cor - 2,5 YR 2,5/4, quando úmido, e 2,5 YR3/4, quando seco - e da baixa taxa de reflectância e alta absorção de energia), promove uma rápida transformação da matéria orgânica, podendo ser esta a justificativa para os menores teores de $C$ total encontrados no solo irrigado.

Para o C mineralizável, as maiores diferenças recaíram sobre os agregados mai ores que 0,25 mm, tendo o processo de respiração sido mais intenso para o PS, o que provavel mente ocorreu em virtude das condições de umidade maiores no PI, tornando o ambiente menos favorável à respiração, ou de um efeito antagônico, causado pela otimização das transformações no momento da deposição do material vegetal, acelerando o processo num curto espaço de tempo. Sob condições de campo, o que se observou foi que a cultura que antecedeu a coleta das amostras para o PI era constituída de leguminosas, enquanto, para o PS, de gramíneas. Assim, observou-se que o material vegetal oriundo de leguminosas foi de mais rápida decomposição que as gramíneas.

\section{Carbono orgânico lábil e carbono solúvel em água}

Para o C orgânico lábil (CL), os valores foram menores no solo sob plantio direto irrigado e de sequeiro, quando comparados aos do sol o sob floresta nativa ( $F N)$, até às profundidades de $0,10-0,20 \mathrm{~m} \mathrm{e}$ $0,20-0,30 \mathrm{~m}$, para os agregados maiores e menores que $0,25 \mathrm{~mm}$, respectivamente (Quadro 7). De acordo com Medeiros \& Mendonça (1994), essa fração orgânica está associada aos cátions trocáveis na superfície de troca do solo. Assim, os resultados de formas de $C$ estão diretamente relacionados com os resultados de CTC efetiva etotal dos sol os estudados, onde o sol o sob mata apresenta os maiores valores.

Por causa de sua rápida assimilação como substrato, a concentração de C solúvel no solo mostrou-se muito baixa, representando menos que $1 \%$ do C total (Killham, 1994). Essa relação variou de 0,09 a $0,23 \%$, para o sol o sob plantio direto e sob floresta nativa, respectivamente.

Para o C solúvel em água (CS), quando se contrastou o PD com a floresta nativa, observou-se que este último apresentou maiores valores tanto para agregados maiores quanto para menores que $0,25 \mathrm{~mm}$, nas profundidades de 0,00-0,20 m e 0,00$0,30 \mathrm{~m}$, respectivamente (Quadro 7). Num ecossistema

Quadro 6. Contrastes entre plantio direto irrigado (PI) e plantio direto de sequeiro (PS) para carbono orgânico total (CT), carbono mineralizável (CM) e carbono da bi omassa microbiana (CB) em agregados maiores e menores que $0,25 \mathrm{~mm}$, respectivamente (1 e 2), de um Latossolo Vermelho E utroférrico Típico, em cinco profundidades

\begin{tabular}{|c|c|c|c|c|c|c|c|}
\hline Tratamento & Profundidade & CT1 & CT2 & CM1 & CM2 & CB1 & CB2 \\
\hline & $\mathrm{m}$ & \multicolumn{2}{|c|}{$\mathrm{mg} \mathrm{kg}^{-1}$} & \multicolumn{2}{|c|}{$\ldots \mathrm{mg} \mathrm{kg}^{-1} \mathrm{de} \mathrm{CO}_{2}$} & $\longrightarrow$ & 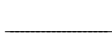 \\
\hline & $\begin{array}{l}0,00-0,05 \\
0,05-0,10\end{array}$ & $\begin{array}{l}-2.500^{\mathrm{NS}} \\
-5.300^{\mathrm{NS}}\end{array}$ & $\begin{array}{r}2.750^{N S} \\
-10.500^{N S}\end{array}$ & $\begin{array}{l}-0,115^{*} \\
-0,115^{*}\end{array}$ & $\begin{array}{l}-0,156^{\circ} \\
-0,098^{\mathrm{NS}}\end{array}$ & $\begin{array}{r}-7,49^{\mathrm{NS}} \\
3,67^{\mathrm{NS}}\end{array}$ & $\begin{array}{r}22,33^{\mathrm{NS}} \\
8,27^{\mathrm{NS}}\end{array}$ \\
\hline PI vs PS & $\begin{array}{l}0,10-0,20 \\
0,20-0,30 \\
0,00-0,05\end{array}$ & $\begin{array}{l}-2.800^{\mathrm{NS}} \\
1.300^{\mathrm{NS}} \\
-2.800^{\mathrm{NS}}\end{array}$ & $\begin{array}{r}2.500 \text { NS } \\
-2.400^{N S} \\
-5.900^{N S}\end{array}$ & $\begin{array}{c}-0,220^{* *} \\
-0,114^{*} \\
0,039^{N S}\end{array}$ & $\begin{array}{l}-0,126^{N S} \\
-0,126^{N S} \\
-0,012^{\text {NS }}\end{array}$ & $-45,84 \mathrm{NS}$ & $3,59 \mathrm{NS}$ \\
\hline
\end{tabular}

NS, ${ }^{\circ}, * e^{* *}$ : não-significativo e significativo a 10,5 e $1 \%$, respectivamente. 
demata, a fontedesubstâncias orgânicas solúveis éa deposiçãonatural deresíduos de plantas, quealcança o solo em forma de fol has, galhos eoutros fragmentos orgânicos, bem como substâncias orgânicas derivadas da decomposição de raízes (Pohlman \& Mccoll, 1988). Assim, os maiores índices de CS encontrados no solo sob floresta nativa se devem, provavel mente, ao maior incremento e à qualidade das substâncias orgânicas no ambiente natural .

Outro fator que pode estar contribuindo para ocorrência de tal fato é que essa fração orgânica é de grande variação temporal e espacial (Zech et al., 1994). No sistema biogeográfico do cerrado, no mês deagosto, época em queforam col etadas as amostras, notou-se uma grande deposição de resíduos vegetais decorrentes da estação seca, sobretudo nos ambientes naturais, o que certamente contribuiu para os maiores valores de CS encontrados no solo sob floresta nativa. O resultado pode ser também similar à constatação de Harris \& Safford (1996) de que há aumento na produção do $C$ solúvel no solo, para os materiais recentemente depositados. Espera-se que o solo sob floresta nativa esteja sempre recebendo novos materiais vegetais, o que mantém sua qualidade.
Para o contraste entre solo sob plantio direto irrigado e plantio direto de sequeiro (PI vs PS), não foi observada diferença significativa em termos de matéria orgânica lábil e $C$ solúvel em água (Quadro 8), provavelmente, por serem as plantas cultivadas basicamente homogêneas.

\section{CONCLUSÕES}

1. O plantio direto não foi capaz de restabelecer os níveis de matéria orgânica do solo e favoreceu a compactação ou adensamento dos sol os estudados.

2. O sistema de plantio direto de sequeiro não acarretou variações significativas nos teores de carbono orgânicototal e nas formas mais dinâmicas de carbono, quando comparado ao sistema de plantio direto irrigado.

3. O C da biomassa microbiana e a matéria orgânica lábil mostraram bom potencial para estudos de ciclagem de nutrientes e para se inferir sobre a agregação, indicando modificações relacionadas com a estruturação do solo.

Quadro 7. Contrastes entre plantio direto (PD) e solo sob floresta nativa (FN) para carbono orgânico lábil (CL) e carbono solúvel em água (CS), para agregados maiores e menores que 0,25 mm, respectivamente (1 e 2), de um Latossolo Vermelho Eutroférrico Típico, em cinco profundidades

\begin{tabular}{cccccc}
\hline \multirow{2}{*}{ Tratamento } & Profundidade & CL1 & CL2 & CS1 & CS2 \\
\hline & $\mathrm{m}$ & & & \\
\cline { 2 - 6 } & $0,00-0,05$ & $-147,32^{* *}$ & $-192,37^{* *}$ & $-120,03^{* *}$ & $-99,78^{* *}$ \\
& $0,05-0,10$ & $-99,38^{* *}$ & $-114,57^{* *}$ & $-47,92^{*}$ & $-73,50^{* *}$ \\
PD\# vs FN & $0,10-0,20$ & $-74,33^{* *}$ & $-95,35^{* *}$ & $-38,88^{\circ}$ & $-40,03^{* *}$ \\
& $0,20-0,30$ & $-20,33^{\mathrm{NS}}$ & $-28,78^{\circ}$ & $-12,25^{\mathrm{NS}}$ & $-28,12^{*}$ \\
& $0,00-0,05$ & $-1,38^{\mathrm{NS}}$ & $-19,37^{\mathrm{NS}}$ & $-13,08^{\mathrm{NS}}$ & $-9,13^{\mathrm{NS}}$ \\
\hline
\end{tabular}

$\mathrm{NS}^{\circ}{ }^{*}$ * e **: não-significativo e significativo a 10, 5 e $1 \%$, respectivamente. ${ }^{*}=$ plantio direto sob irrigação e de sequeiro.

Quadro 8. Contrastes entre plantio direto irrigado (PI) e plantio direto de sequeiro (PS) para carbono orgânico lábil (CL) e carbono solúvel em água (CS), para agregados maiores e menores que 0,25 mm, respectivamente (1 e 2), de um Latossolo Vermelho Eutroférrico Típico, em cinco profundidades

\begin{tabular}{|c|c|c|c|c|c|}
\hline Tratamento & Profundidade & CL1 & CL2 & CS1 & CS2 \\
\hline & $\mathrm{m}$ & \multicolumn{4}{|c|}{$\longrightarrow \mathrm{g} \mathrm{kg}^{-1}$} \\
\hline & $\begin{array}{l}0,00-0,05 \\
0,05-0,10\end{array}$ & $\begin{array}{l}-7,50^{\text {NS }} \\
-4,77^{\text {NS }}\end{array}$ & $\begin{array}{r}-19,53^{\mathrm{NS}} \\
-3,87^{\mathrm{NS}}\end{array}$ & $\begin{array}{r}-3,67 \mathrm{NS} \\
6,57^{\mathrm{NS}}\end{array}$ & $\begin{array}{l}-7,03^{\mathrm{NS}} \\
-3,40^{\mathrm{NS}}\end{array}$ \\
\hline PI vs PS & $\begin{array}{l}0,10-0,20 \\
0,20-0,30 \\
0,00-0,05\end{array}$ & $\begin{array}{r}-5,87^{\mathrm{NS}} \\
-29,53^{\mathrm{NS}} \\
-0,57^{\mathrm{NS}}\end{array}$ & $\begin{array}{l}-16,37^{N S} \\
-18,03^{N S} \\
-24,67^{N S}\end{array}$ & $\begin{array}{r}2,23^{\mathrm{NS}} \\
-7,83^{\mathrm{NS}} \\
-12,70^{\mathrm{NS}}\end{array}$ & $\begin{array}{l}-9,13^{\mathrm{NS}} \\
-6,23^{\mathrm{NS}} \\
-9,60^{\mathrm{NS}}\end{array}$ \\
\hline
\end{tabular}

NS: não-significativo. ${ }^{\#}=$ plantio direto sob irrigação e de sequeiro. 


\section{LITERATURA CITADA:}

ALVAREZ, R. \& LAVADO, R.S. Climatic, organic matter and clay content relationship in the Pampa and Chaco soils, Argentina. Geoderma, 83:127-141,1998.

ANDERSON, D.W.; SAGGAR, S.; BETTANY, J .R. \& STEWART, J.W.B. Particle size fractions and their use in studies of soil organic matter. I. The nature and distribution of forms of carbon, nitrogen, an sulfur. Soil Sci. Soc. Am. J ., 45:767$772,1981$.

ANGERS, D.A.; BOLINDER, M.A.; CARTER, M.R.; GREGORICH，E.G.; DRURY, C.F.; LIANG, B.C.; VORONEY, R.P.; SIMARD, R.R.; DONALD, R.G.; BEYAERT, R.P. \& MARTEL, J . I mpact of tillage practices on organic carbon and nitrogen storage in cool, humid soils of eastern Canada. Soil Till. Res., 41:191-201, 1997.

ARCA, M.N. \& WEED, S.B. Soil aggregation and porosity in relation to contents of free iron oxide and clay. Soil Sci. 101:164-170, 1966.

BARBOSA, A.S. Sistema biogeográfico do cerrado: alguns el ementos para sua caracterização. Contribuições 3. Goiânia, ITS/UCG, 1996. p.14-18.

BARTLETT, R.J . \& ROSS, D.N. Colorimetric determination of oxidizable carbon in acid soil solutions. Soil Sci. Soc. Am. J ., 52:1191-1192, 1988.

BROOKES, P.C.; LANDMAN, A.; PRUDEN, G. \& J NKINSON, D.S. Chloroform fumigation and the release of soil nitrogen: a rapid direct extraction method to measure microbial biomass nitrogen in soil. Soil Biol. Biochem., 17:837-842, 1985.

COLOZZI-FILHO A.; BALOTA, E.L. \& ANDRADE, D.S. Microrganismos e processos biológicos no sistema plantio direto. In: SIQUEIRA, J.O.; MOREIRA, F.M.S.; LOPES.A.S.; GUILHERME, L.R.G.; FAQUIN, V.; FURTINI NETO, A.E. \& CARVALHO, J.C., eds. Interrelação fertilidade, biologia do solo e nutrição de plantas, Lavras, Viçosa:SBCS, Lavras, Universidade Federal de Lavras, 1999. p.487-508.

DEFELIPO, B.V. \& RIBEIRO, A.C. Análise química do solo: metodologia. Viçosa, UniversidadeF ederal deViçosa, 1981. 15p.

DUXBURY, J .M.; SMITH, M.S.; DORAN, J .W.; J ORDAN, C.; SZOTT, L. \& VANCE, E. Soil organic matter as a source and a sink of plants nutrients. In: COLEMAN, D.C.; OADES, J .M. \& VEHARA, G., eds. Dynamics of soil organic matter in tropical ecosystems. Hawaii, University of Hawaii Press, 1989. p.33-67.

EDWARDS, A.P. \& BREMNER, J.M. Microaggregates in soils. J. Soil Sci., 18:64-73, 1967.

EMPRESA BRASILEIRA DE PESQUISA AGROPECUÁRIA EMBRAPA. Sistema brasileiro de classificação de solos. Brasília, 1999. 412p.

EMPRESA BRASILEIRA DE PESQUISA AGROPECUÁRIA EMBRAPA. Serviço Nacional de Levantamento e Conservação de Solos. Manual de métodos de análise de solo. Rio de J aneiro, Ministério da Agricultura, 1997. Não paginado.
EVANS J r., A.; ZELAZNY, L.W. \& ZIPPER, C.E. Solution parameters influencing dissolved organic carbon levels in three forest soils. Soil Sci. Soc. Am. J ., 52:1789-1792, 1988.

GAMA-RODRIGUES, E.F.; GUERRA, J .G.M.; ALMEIDA, D.L. $\&$ DE-POLLI, H._Biomassa microbiana de carbono de sol os de I taguaí (RJ ). Comparação entre os métodos fumigaçãoincubação e fumigação-extração. R. Bras. Ci. Solo, 18:427432, 1994.

GOEDERT, W.J . Management of the cerrado soils of Brazil: a review. J. Soil Sci., 34:405-428, 1983.

GREENLAND, D.J. \& OADES, J.M. Saccharides. In: GREENLAND, D.J . \& OADES, J .M. Soil components. New York, Springer-Verlag, 1975. v.1. p.213-261.

GUGGENBERGER, G. \& ZECH, W. Dissolved organic carbon in forest floor leachates: simple degradation products or humic substances? Sci. Total Environ., 152:37-47, 1994.

GUPTA, V.V.S.R. \& GERMIDA, J J . Distribution of microbial biomass and its activity in different soil aggregate size classes as affected by cultivation. Soil Biol. Biochem., 120:777-786, 1988.

HARRIS, M.M. \& SAFFORD, L.O. Effects of season and four tree species on soluble carbon content in fresh and decomposing litter of temperate forests. Soil Sci., 161:130135, 1996.

J UCKSCH, I. Calagem e dispersão de argila em amostras de um Latossolo Vermelho Escuro. Viçosa, Universidade Federal de Viçosa, 1987. 37p. (Tese de Mestrado)

KER, J.C.; PEREIRA, N.R. \& CARVALHO J UNIOR, W.C. Cerrado: solos, aptidão e potencialidade agrícola. In: SIMPÓSIO SOBRE MANEJ O E CONSERVAÇÃO DO SOLO NO CERRADO, 1992, Goiânia. Anais. Goiânia, 1992. $167 p$.

KILLHAM, K. Soil ecology. Cambridge, Cambridge University Press, 1994. 242p

MEDEIROS, M. \& MENDONÇA, E.S. Labile organic matter and its relation with exchangeable el ements of oxisols with different mineralogy, texture and organic matter content. WORLD CONGRESS OF SOIL SCIENCE, 15., Acapulco, 1994. Anais. Acapulco, International Society of Soil Science and the Mexican Society of Soil Science, 1994. v.3b. (Commission II: Poster sessions)

MUZILLI, O. Influência do sistema plantio direto, comparado ao convencional, sobre a fertilidade da camada arável do solo. R. Bras. Ci. Solo, 7:95-102, 1983.

PAUSTIAN, K.; COLLINS, H.P. \& PAUL, E.A. Management controls on soil carbon. In: PAUL, E.A.; PAUSTIAN, K.; ELLIOT, E.T. \& COLE, C.V., eds. Soil organic matter in temperate agroecosystems, Long-term experiments in North America. Boca Raton, CRC Press, 1997. p15-49.

POHLMAN, A.A. \& MCCOLL, J .G. Soluble organics from forest litter and their role in metal dissolution. Soil Sci. Soc. Am. J., 52:265-271, 1988.

RESCK, D.V.S.; PEREIRA, J \& \& SILVA, J.E. Dinâmica da matéria orgânica na região dos cerrados. Planaltina, Empresa Brasileira de Pesquisa Agropecuária - Centro de Pesquisa Agropecuária do Cerrado, 1991. 22p. 
SÁ, J .C.M Manejo da fertilidade do solo em plantio direto. In: Plantio direto no Brasil. Castro, EMBRAPA-CNPT, 1993. p.37-60.

SÁ, J.C.M. Manejo da fertilidade do solo no sistema plantio direto. In: SIQUEIRA, J .O.; MOREIRA, F.M.S.; LOPES, A.S.; GUILHERME, L.R.G.; FAQUIN, V.; FURTINI NETO, A.E. \& CARVALHO, J.G., eds. Inter-relação fertilidade, biologia do solo e nutrição de plantas. Viçosa, Sociedade Brasileira de Ciência doSolo, Lavras, Universidade F ederal de Lavras, 1999. 818p.

SIDIRAS, N. \& PAVAN, M.A. Influência do sistema de manejo do solo no seu nível de fertilidade. R. Bras. Ci. Solo, 9:249254, 1985.

SIQUEIRA, J .O. Biologia do solo. Lavras, Escola Superior de Agricultura de Lavras/FAEPE, 1993. 230p.

STEVENSON, F.J. Humus chemistry. 2.ed. New York, J ohn Wiley \& Sons, 1994. 496p.
THENG, B.K.G.; TATE, K.R. \& SOLLINS, P. Constituents of organic matter in temperate and tropical soil. In: COLEMAN, D.C.; OADES, J .M. \& UEHARA, G. Dynamics of organic matter in tropical ecosystems. Honolulu, University of Hawaii, 1989. p.5-31.

TISDALL, J .M. \& OADES, J .M. Organic matter and waterstable aggregates in soil. J. Soil Sci., 33:141-163, 1982.

ZECH, W.; GUGGENBERGER, G. \& SCHULTEN, H.R. Budgets and chemistry of dissolved organic carbon in forest soils: effects of anthropogenic soil acidification. Sci. Total Environ., 152:49-62, 1994.

ZIBILSKE, L.M. Carbon mineralization. In: WEAVER, R.W.; ANGLE, S.; BOTTOMLEY, P.; BEZDICEK, D.; SMITH, S.; TABATABAI, A. \& WOLLUM, A. Methods of soil analysis. Part 2. Microbiological and biochemical properties. Madison, Soil Science Society of America, 1994. p.835-863. (Book Series, 5) 
M.E.C. ROSA et al. 